\title{
Detection and Characterization of Heavy-Metal-Free (HMF) Gunshot Residues Using CL, EDS and Raman Together with XRD
}

\author{
Ken Mason ${ }^{1}$ and Richard Wuhrer ${ }^{2}$ \\ 1. Eastern Analytical sprl, Szőce, Hungary. \\ 2. Western Sydney University Advanced Materials Characterisation Facility, NSW, Australia.
}

In recent years, heavy metal free (HMF) cartridge primers have been developed to reduce health risks to occupational (police) and sports shooters from lead $(\mathrm{Pb})$ in particular. Most HMF manufacturers have primer formulations that divide into four basic groups - 1) TiZn, 2) Sr, 3) $\mathrm{Zr}$ and 4) NaAlSiK. Gunshot residues (GSR) from suspects, clothing or the environment have traditionally been detected using SEM and EDS. However, HMF residues are not so easily detectible by these means and present a challenge to investigators. Especially difficult to detect are residues from NaAlSiK primers due to its low average atomic number.

A study was made to determine ways to detect NaAlSiK residues from S\&B NONTOX ${ }^{\mathrm{TM}}$ and CBC CleanRange ${ }^{\mathrm{TM}}$ ammunition. An examination of particles from the cartridge cases and shooter were made and it was found that particles from both ammunition types exhibited cathodoluminescence (CL) when scanned by an electron beam in a SEM. It was also found that particles exhibiting cathodoluminescence, had morphologies consistent with being produced in a high-temperature explosive environment.

Normally, residues from $\mathrm{Pb}$ primers (SINOXID) are detected in a SEM on the basis of the fact that their average atomic number and their chemistry are significantly different from environmentally occurring particles. NaAlSiK primer residues do not have this characteristic but, if they can be detected by their CL emission, the same search strategy, as is employed with SINXOID (using the backscatter signal), could be used with the CL signal. This automatic search mechanism was implemented and found successful.

However the question remained as to how the CL signal was being produced and why other particles that are not GSR, but that show CL, would not be erroneously detected by the system. A study was then made to show what chemical characteristics of candidate GSR particles show both CL and morphology. Particles from the cartridge case were also studied to determine whether the residues were amorphous or crystalline and to see if Raman examination could show what phase(s) these particle were.

In addition, X-ray diffraction (XRD) was used to see if the particles' pattern could be matched to a known phase. Similarly, a soft UV light source was used to see if the particles of interest glowed under $365 \mathrm{~nm}$ UV. Preliminary results reveal the CL emitting GSR particles have very similar XRD patterns to crystalline glasses and feldspars such as microcline, sanidine and potassium based feldspars. Major problem was this work had to be done with cartridge case scrapings as particles from shooters, although emitting CL, were too small $(<10 \mu \mathrm{m})$ for the XRD and Raman studies. However, detection of shooter particles was demonstrated using CL and EDS.

The system designed and built has a cathodoluminescence panchromatic detector coupled to a CCD UVVIS-NIR spectrometer which allowed detailed imaging and measurement of the particles' light emissions and CL topography (Figure 1). This apparatus was specifically designed for detection of these 
particles. Originally the SED detector on the Phenom instrument had been modified to detect CL by disconnecting the high tension (HT) bias power and removing the YAG scintillator and just leaving the light pipe that goes to the photomultiplier tube (PMT). This paper will discuss modifications that have been completed to obtain a CL detector and the subsequent implantation of a CL spectroscopy system. The preliminary results obtained from the CL detector from investigating GSR particles (Figure 2) will be presented.

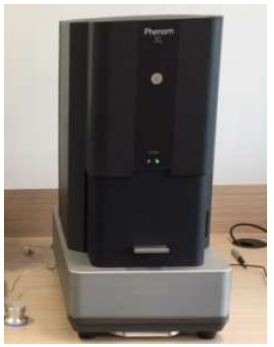

a.

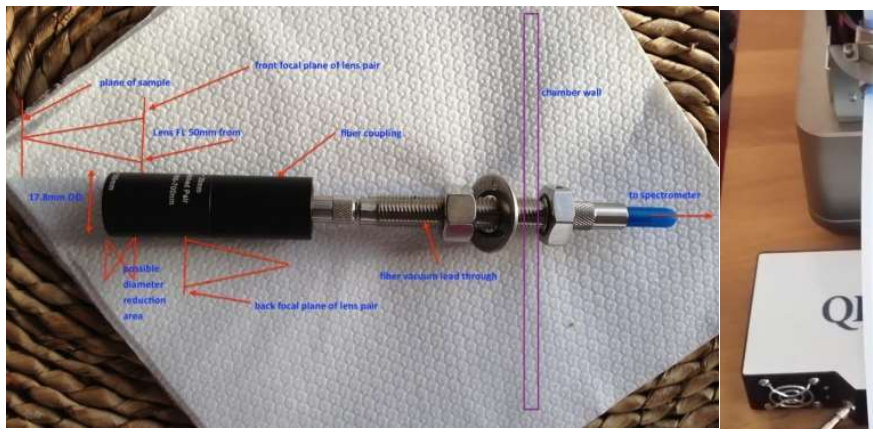

d.

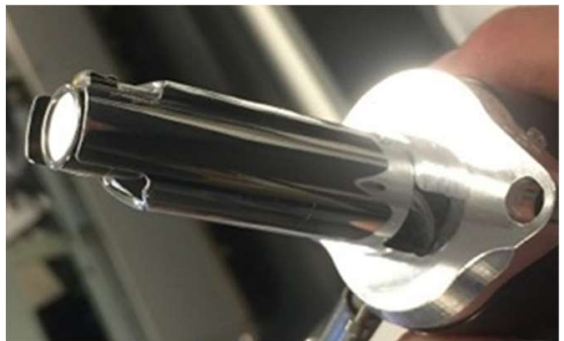

b.

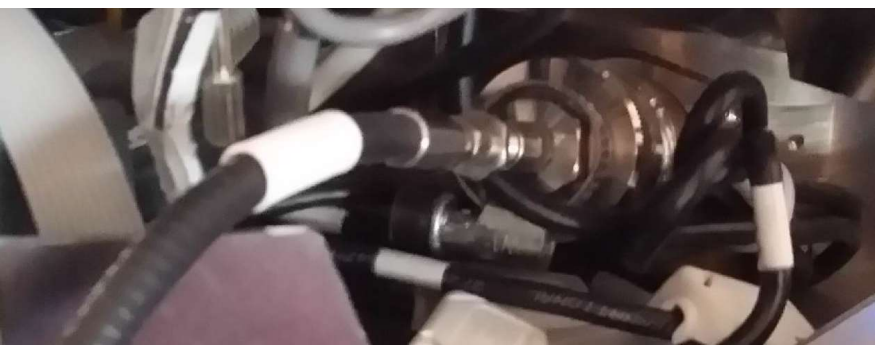

C.

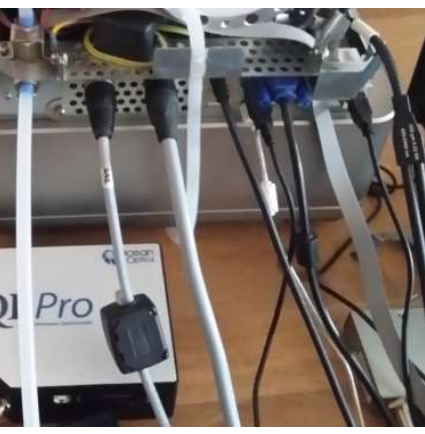

e.

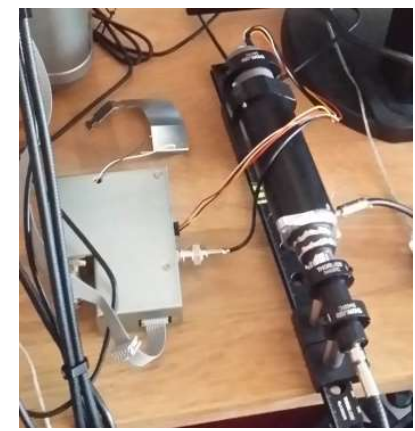

f.

Figure 1. a) Typical Phenom XL with SDD, X-ray mapping (XRM) and GSR analysis, b) SED detector before modification into a CL detector, c) cathodoluminescence light capture system incorporated into SED housing, d) design of cathodoluminescence light capture system, e) spectrometer and f) modified photomultiplier mounted with fiber connection to " $\mathrm{c}$ "

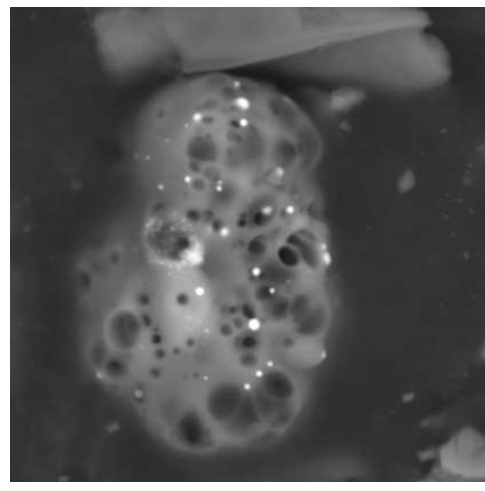

a.

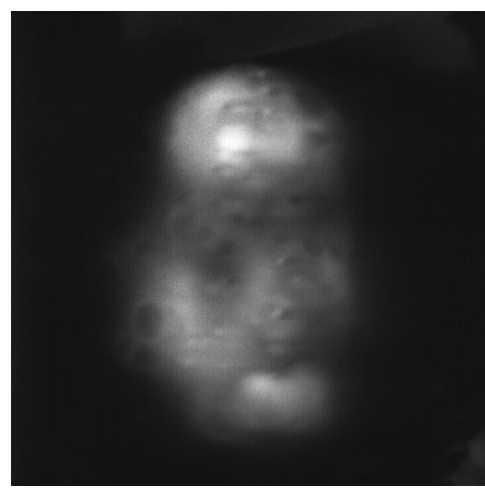

b.

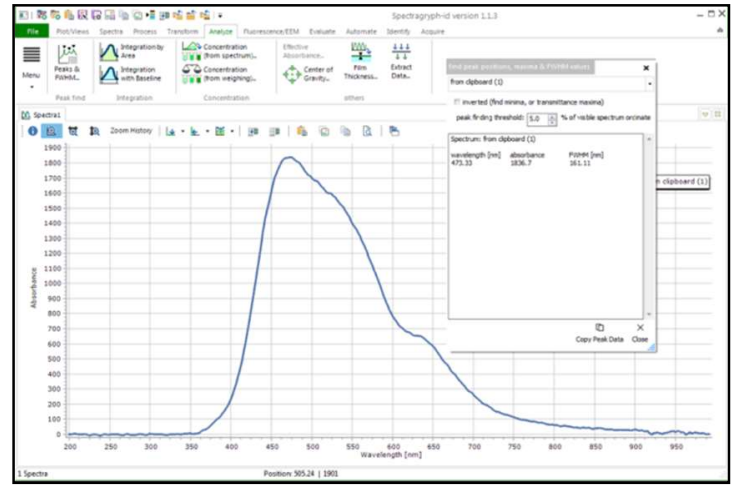

c.

Figure 2. A large $100 \mu \mathrm{m}$ composite S\&B Nontox GSR particle, showing morphology and chemistry of GSR. a) BSE image, b) CL image and c) spectroscopy graph of the light from this particle. 